\title{
Medial collateral ligament reconstruction using bone-patellar tendon-bone allograft for chronic medial knee instability combined with multi-ligament injuries: a new technique
}

\author{
Xiaozuo Zheng ${ }^{1,2^{*}}$, Tong $\mathrm{Li}^{1,2}$, Juan Wang ${ }^{1,2}$, Jiangtao Dong ${ }^{1,2}$ and Shijun Gao ${ }^{1,2^{*}}$
}

\begin{abstract}
Background: The medial collateral ligament (MCL) is the main static stabilizer of the medial knee. The surgical treatment was recommended in cases with serious medial collateral ligament insufficiency combined with multi-ligament injuries and chronic symptomatic medial instability. Several surgical techniques have been described for the MCL reconstruction, while potential problems including donor site morbidity, complicated procedure, and high risk of femoral tunnel collision were reported. In order to minimize such potential limitations, we describe a new medial reconstruction technique for MCL injury using bone-patellar tendon-bone (BPTB) allograft.

Methods: A longitudinal incision at the medial knee was made. The centers of femoral and tibial attachments were gained through repeated isometricity test. Then, the bone grooves were made around the femoral and tibial centers. The appropriate BPTB allograft was selected, and both ends were trimmed. The prepared bone blocks were embedded into the grooves and fixed with cancellous screws. The programmed rehabilitation exercises were performed after the operation.

Results: A strong graft and bone-to-bone healing on both femoral and tibial attachment sites were obtained, and femoral tunnel collision during multi-ligament reconstruction was avoided. Satisfactory valgus and rotatory stability were gained.

Conclusions: This novel MCL reconstruction technique using BPTB allograft can be safely performed, and the clinical outcome was favorable with satisfactory valgus and rotatory stability. More cases and additional follow-up results are needed to verify the overall effect of this technique.
\end{abstract}

Keywords: Knee, Medial collateral ligament, Reconstruction, Technique

\section{Background}

The medial collateral ligament (MCL) is the main static stabilizing structure against valgus and rotation of the knee. It is a broad, flat, and long ligamentous tissue, which originates around the medial femoral condyle and inserts on the proximal medial tibia. Most MCL injuries could be treated nonoperatively with good clinical

\footnotetext{
*Correspondence: xiaozuo_zheng@163.com; 13595933@qq.com

'Department of Orthopedics, Third Hospital of Hebei Medical University, 139

Ziqiang Road, Shijiazhuang 050051, Hebei, People's Republic of China

Full list of author information is available at the end of the article
}

outcomes because of the strong healing capacity $[1,2]$. However, surgical treatment was recommended in cases with serious MCL insufficiency combined with multiligament injuries and chronic symptomatic medial instability [3-5]. In chronic cases with serious medial knee instability, there is little chance that the MCL will heal well and restore valgus stability [6]. Therefore, surgical treatment would be necessary.

Several surgical techniques have been described for the MCL reconstruction, such as direct repair [7, 8], proximal advancement of MCL [9], isolated MCL reconstruction 
$[10,11]$, and anatomical MCL reconstruction using a double-bundle technique $[4,12]$. However, potential problems with these surgical techniques were reported. Yoshiya et al. argued that the autogenous semitendinosus and gracilis tendons graft harvest in the MCL reconstructive procedure may result in a loss of function of the pes anserinus tendons which serve as secondary medial stabilizers [10]. Dong et al. considered that augmented repair was not a good choice for the subacute MCL injury, and simple repair may not be as reliable as surgical reconstruction [8]. Moreover, in cases of simultaneous posterior cruciate ligament (PCL)-MCL reconstruction, two tunnels are needed to be created in the same condyle and close to each other. In this circumstance, high risk of tunnel collision was found during the surgical procedure [13].

In order to minimize such potential limitations, we describe a simple reconstruction procedure of MCL for chronic medial knee instability using bone-patellar tendon-bone allograft. The rationale of this technique is to obtain a strong graft and bone-to-bone healing on both femoral and tibial attachment sites and to avoid femoral tunnel collision during multi-ligament reconstruction.

\section{Surgical procedure}

A 50-year-old man suffered a traumatic accident and was diagnosed with anterior cruciate ligament (ACL), PCL, and grade III MCL injuries of the right knee. The patient underwent simultaneous ACL and PCL reconstruction, combined with MCL anatomic repair at the time of surgery as acute injuries. While residual medial laxity was still obvious 3 months after direct repair and we believed that a reconstructive procedure was needed, with the patient under general or epidural anesthesia, the physical examination was performed to confirm MCL laxity that required reconstruction. A routine diagnostic arthroscopic evaluation was carried out and intra-articular damages were treated. As expected, continuity was seen on any of the reconstructed grafts, and subjective tension of either graft with a probe was marked taut.

After the arthroscopic procedure, the following reconstruction steps were carried out. A longitudinal incision was made from $1 \mathrm{~cm}$ above the medial femoral epicondyle to the insertion of the pes anserinus. (In this case, the incision previously made for repairing MCL was used). The medial structures of the knee were assessed under direct visualization. The medial femoral epicondyle and pes anserinus were identified as the landmarks of the femoral and tibial attachments of superficial MCL. The repair began from the deepest structures to the most superficial structures (Fig. 1). Interrupted absorbable suture method was used for repairing, if needed.

A 2-mm guide pin was drilled at the center of the femoral attachment of the MCL, which was located 3-5 mm proximal and posterior to the medial epicondyle. The estimated tibial insertion was located about $4.5 \mathrm{~cm}$ below the tibial plateau. An isometric test was performed through a $0-90^{\circ} \mathrm{knee}$ motion. The femoral and tibial insertion points were modified until satisfactory isometricity; a less than 2-mm length change during knee movement was gained. Once the isometric points were determined, the bone grooves were made around the centers of the femoral and tibial attachments. The femoral portion was about $10 \mathrm{~mm}$ in length, $10 \mathrm{~mm}$ in width, and $3 \mathrm{~mm}$ in depth. The tibial potion was about $20 \mathrm{~mm}$ in length, $10 \mathrm{~mm}$ in width, and $3 \mathrm{~mm}$ in depth (Fig. 2).

The length between the femoral and tibial grooves was measured with the knee placed at $30^{\circ}$ of flexion with varus stress and neutral rotation. The appropriate bone-patellar tendon-bone (BPTB) allograft was selected according to the length of measurement. Both ends were trimmed into rectangular shapes to match the bone grooves (Fig. 3). Then, the femoral and tibial bone blocks were embedded into the grooves and fixed with cancellous screws, with

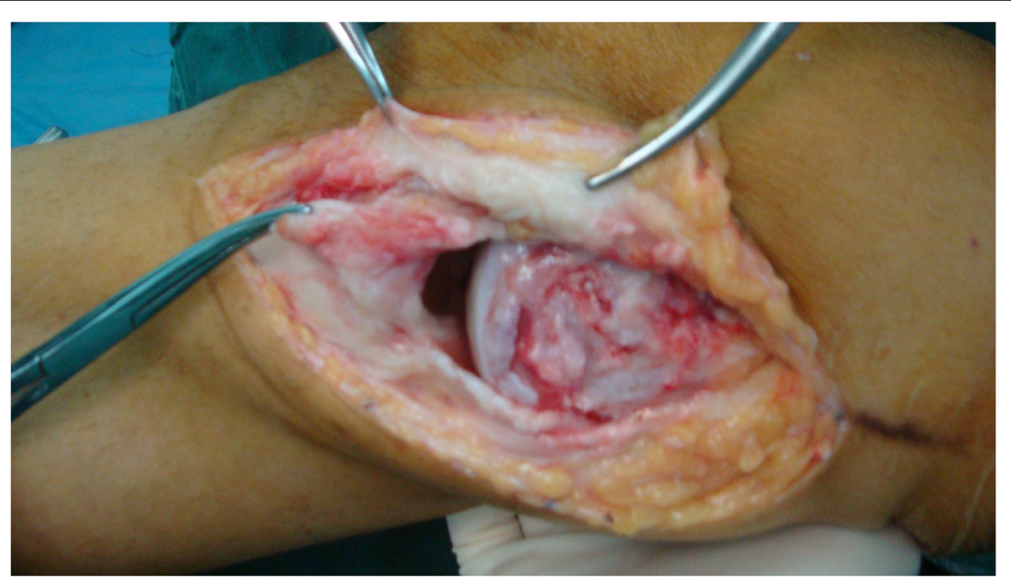

Fig. 1 A longitudinal incision was made, and the severity of MCL damage was evaluated 


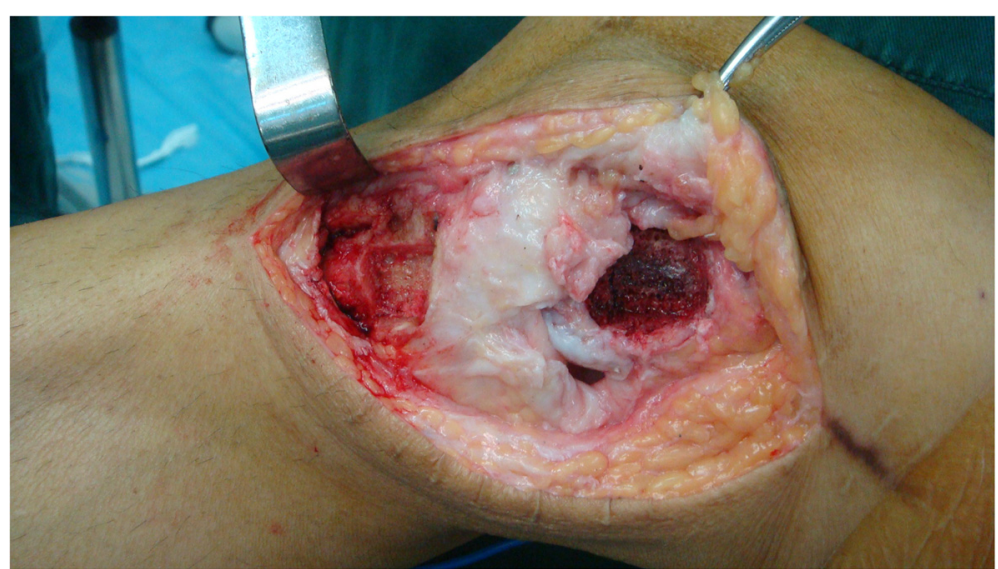

Fig. 2 The bone grooves were made around the centers of the femoral and tibial attachments after satisfactory isometricity was gained through repeated modification

the knee at $30^{\circ}$ of flexion with varus stress and neutral rotation (Fig. 4). If possible, the torn posterior oblique ligament (POL) was identified and repaired with interrupted absorbable sutures. The anterior aspect of it was sutured to the posterior region of the reconstructed MCL. After all procedures were completed, a gentle valgus test was performed to ensure that the stability of the MCL reconstruction was adequate.

Postoperatively, a long hinged brace locked in extension was used for 2 weeks. The patient was encouraged to do isotonic exercise for quadriceps and hamstrings $48 \mathrm{~h}$ after the surgery. For isolated MCL reconstruction, knee motion of $30^{\circ}-90^{\circ}$ in the brace was allowed from four to six postoperative weeks. A goal of $120^{\circ}$ flexion was expected at 8 weeks postoperatively. Partial and full weight-bearing exercises were allowed 6 to 12 weeks after surgery, and the brace should be weaned off gradually. Free activity was allowed after 3 months, and return to sports was not permitted until 6 months postoperatively.
For combined MCL-ACL reconstruction, a similar rehabilitation plan was applied, but return to sports should be delayed to at least 9 months after the operation. For combined MCL-PCL reconstruction, more restrictive rehabilitation plan was applied. The brace wearing time should last for at least 3 months. Partial weight-bearing exercise should be delayed to 8 weeks postoperatively, and the brace could not be weaned off until 12 weeks postoperatively. Return to sports was not allowed until 12 months after the surgery.

\section{Discussion}

Most of the grafts used for MCL reconstruction are tendon-like tissue, and the femoral and tibial attachments are point-like which were fixed with interference screws. However, the anatomical studies have demonstrated that the superficial MCL is a broad, flat, and long ligamentous tissue and almost composed of parallel fibers [14, 15]. The femoral and tibial attachments were broad-based, with

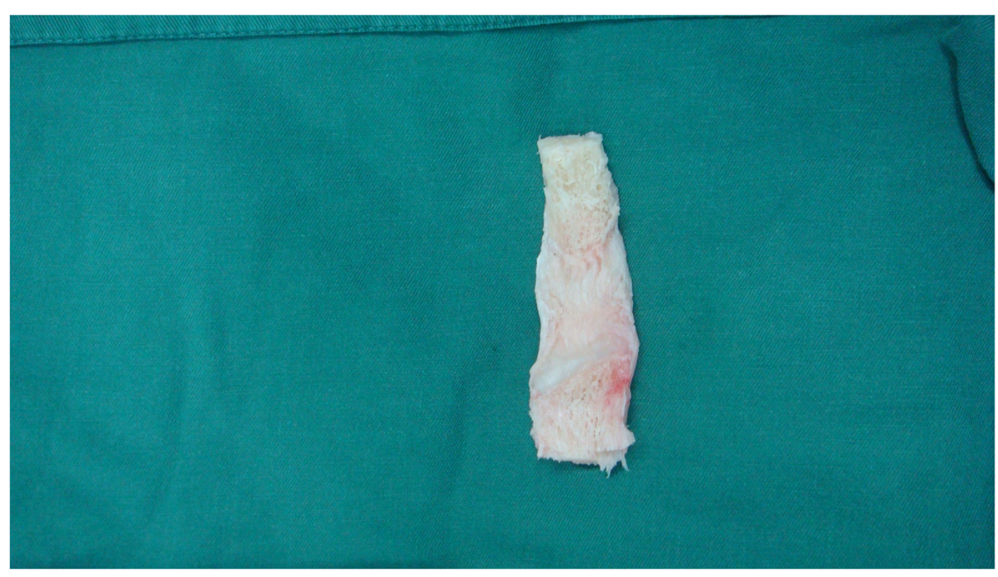

Fig. 3 Bone-patellar tendon-bone (BPTB) allograft was selected, and both ends were trimmed to match the bone grooves 


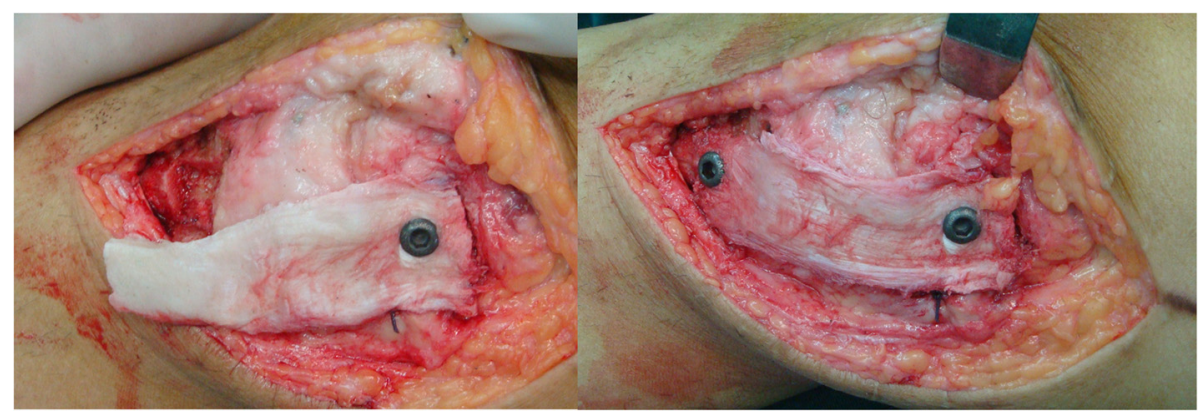

Fig. 4 The femoral and tibial bone blocks were embedded into the grooves and fixed with cancellous screws

areas 79.7 and $348.6 \mathrm{~mm}^{2}$, respectively. The width of the superficial MCL was about 10 to $17 \mathrm{~mm}$ from the proximal and distal ends to the middle part [15]. We do not think the tendon-like graft could provide sufficient strength compared with uninjured MCL in the early period of ligament reconstruction, although biomechanical studies are needed to prove our hypothesis. In our technique, BPTB was used as graft for reconstruction of MCL. The first advantage was that the BPTB graft could reproduce the shape of superficial MCL more anatomically because it was also a broad, flat, and long ligamentous tissue with two large insertion ends. More importantly, the graft offers sufficient strength for restoring knee kinematics and stability compared with single and thin tendon graft. Secondly, the BPTB graft provided two cancellous bone ends and that allows bone-bone healing at both femoral and tibial attachments, which are more reliable than tendon-bone healing.

Simultaneous MCL-ACL and MCL-PCL injuries are very common. During these multi-ligament reconstruction procedures, two or more femoral tunnels were required for graft placement. In cases of simultaneous MCL-ACL reconstruction, no femoral tunnel collision would happen because of different condyle tunnel placement. While, in cases of simultaneous MCL-PCL reconstruction, high risk of femoral tunnel collision was found because two tunnels were created in the same condyle and close to each other [13], in our surgical procedure, no femoral tunnel was needed for MCL reconstruction procedure. Only a 3-mm-depth bone groove was created under direct vision, and the graft placement was relatively simple. A guide pin was firstly initiated under fluoroscopy to avoid screw-PCL tunnel collision and that allows secure fixation on the femoral end. By taking this technique, we could simultaneously reconstruct $\mathrm{MCL}$ and PCL safely and effectively.

Several double bundle techniques have been described to restore medial knee stability. Many authors suggested superficial MCL reconstruction combined with POL reconstruction for chronic medial laxity. The POL is a condensation of the posteromedial joint capsule located posterior to the superficial MCL [16] and serves as a primary restraint to the rotation and a secondary restraint to valgus translation [4]. While whether concomitant superficial MCL-POL reconstruction could better improve both valgus and rotation stability compared with isolated MCL reconstruction is still debated [17] and the indication for reconstruction of the POL in the treatment of medial knee injuries requires further research, previous study argued that the superficial MCL also played an important role in maintaining rotational stability [8]. It could effectively restore favorable kinematics and stability in the superficial MCL and POL deficient knee by using a broad graft $[6,17]$. From a mechanical view, a quadrangular structure could provide rotatory stability to some extent. We do think this broad quadrangular BPTB graft used in our technique could provide enough tissue and sufficient strength for restoring both valgus and rotational stability. Further biomechanical test and case-series studies are required to evaluate the outcome of this technique.

For medial knee injuries, controversies still exist over that how to choose the best treatment reasonably. It was generally accepted that isolated low-grade, partial medial collateral ligament injuries (grade I and II) could be treated nonoperatively [18]. While the surgery was recommended in cases of complete medial knee injuries (grade III) or with concomitant cruciate ligament injuries [18], some surgeons have recommended MCL reconstruction rather than surgical repair for chronic phase because poor-quality scarring and incomplete healing of the MCL are very difficult to identify and repair of those structures could hardly yield satisfactory results $[7,8,19]$. In addition, in cases of complete medial knee injuries combined with cruciate ligament injuries, MCL reconstruction was more reliable than simple repair to restore knee stability [8]. Thus, this MCL reconstruction technique was recommended in the following situations: (1) chronic grade III MCL injury with symptoms of medial instability, (2) acute medial knee injury combined with cruciate ligament injury that need simultaneous 
reconstruction, and (3) failure of MCL repair or reconstruction that need revision.

Certainly, there are some limitations in our MCL reconstruction technique. Firstly, this new technique may not reconstruct the superficial MCL anatomically because of the attachment sites of the reconstructed MCL depending on the length of BPTB allograft. While the isometric point should be gained through repeated modifications, the medial stability could be restored satisfyingly in knee flexion and extension positions. Secondly, two metallic screws were used to fix the graft. Friction at the skin-screw interface may lead to pain and tenderness in the medial part of the knee. Thus, an additional operation to remove the internal fixation was recommended when the bone healing was achieved. In addition, potential weakness in the present study was that the number of patients was limited, short-term follow-up period, and we also did not evaluate postoperative performance of the patients.

\section{Conclusions}

This novel MCL reconstruction technique using a broad and strong BPTB allograft provides bone-to-bone healing on both the femoral and tibial attachments. More importantly, it could provide satisfactory valgus and rotatory stability and avoid femoral tunnel collision during multi-ligament reconstruction. More cases and additional follow-up results are needed to verify the overall effect of this technique.

\section{Abbreviations}

ACL, anterior cruciate ligament; BPTB, bone-patellar tendon-bone; $\mathrm{MCL}$, medial collateral ligament; $\mathrm{PCL}$, posterior cruciate ligament; $\mathrm{POL}$, posterior oblique ligament

\section{Acknowledgements}

None

\section{Funding}

This work has been supported by Key project of medical science of Hebei province (No. 20160154)

\section{Authors' contributions}

SG performed the surgery and also revised the manuscript. XZ checked the medical records, performed the clinical follow-up, and also drafted the manuscript. TL, JW, and JD have participated in the surgery and contributed to the manuscript preparation. All authors read and approved the final manuscript.

\section{Competing interests}

The authors declare that they have no competing interests.

\section{Consent for publication}

The patient was informed that data concerning his case would be submitted for publication, and he consented. All authors read, approved the final manuscript, and consented to publication.

\section{Ethics approval and consent to participate}

The investigation process was approved by the ethics committee of the Third Hospital of Hebei Medical University.

\section{Author details}

${ }^{1}$ Department of Orthopedics, Third Hospital of Hebei Medical University, 139

Ziqiang Road, Shijiazhuang 050051, Hebei, People's Republic of China.

${ }^{2}$ Orthopaedic Biomechanics Laboratory of Hebei Province, 139 Ziqiang Road,

Shijiazhuang 050051, Hebei, People's Republic of China.

Received: 15 April 2016 Accepted: 1 July 2016

Published online: 22 July 2016

\section{References}

1. Edson CJ. Conservative and postoperative rehabilitation of isolated and combined injuries of the medial collateral ligament. Sports Med Arthrosc. 2006:14:105-10.

2. Miyamoto RG, Bosco JA, Sherman $\mathrm{OH}$. Treatment of medial collateral ligament injuries. J Am Acad Orthop Surg. 2009;17:152-61.

3. Wijdicks CA, Griffith CJ, Johansen S, Engebretsen L, LaPrade RF. Injuries to the medial collateral ligament and associated medial structures of the knee. J Bone Joint Surg Am. 2010;92:1266-80.

4. Liu H, Wang F, Kang H, Chen B, Zhang Y, Ma L. Anatomical reconstruction of the medial collateral ligament and the posterior oblique ligament of the knee. Acta Orthop Belg. 2012;78:400-4.

5. Lind M, Jakobsen BW, Lund B, Hansen MS, Abdallah O, Christiansen SE. Anatomical reconstruction of the medial collateral ligament and posteromedial corner of the knee in patients with chronic medial collateral ligament instability. Am J Sports Med. 2009;37:1116-22.

6. Zhang H, Bai X, Sun Y, Han X. Tibial inlay reconstruction of the medial collateral ligament using Achilles tendon allograft for the treatment of medial instability of the knee. Knee Surg Sports Traumatol Arthrosc. 2014;22:279-84.

7. Canata GL, Chiey A, Leoni T. Surgical technique: does mini-invasive medial collateral ligament and posterior oblique ligament repair restore knee stability in combined chronic medial and ACL injuries? Clin Orthop Relat Res. 2012;470:791-7.

8. Dong J, Wang XF, Men X, Zhu J, Walker GN, Zheng XZ, Gao JB, Chen B, Wang $F$, Zhang $Y$, Gao SJ. Surgical treatment of acute grade III medial collateral ligament injury combined with anterior cruciate ligament injury: anatomic ligament repair versus triangular ligament reconstruction. Arthroscopy. 2015;31:1108-16.

9. Koga H, Muneta T, Yagishita K, Ju YJ, Sekiya I. Surgical management of grade 3 medial knee injuries combined with cruciate ligament injuries. Knee Surg Sports Traumatol Arthrosc. 2012;20:88-94.

10. Yoshiya S, Kuroda R, Mizuno K, Yamamoto T, Kurosaka M. Medial collateral ligament reconstruction using autogenous hamstring tendons: technique and results in initial cases. Am J Sports Med. 2005;33:1380-85.

11. Marx RG, Hetsroni I. Surgical technique: medial collateral ligament reconstruction using Achilles allograft for combined knee ligament injury. Clin Orthop Relat Res. 2012:470:798-805.

12. Borden PS, Kantaras AT, Caborn DN. Medial collateral ligament reconstruction with allograft using a double-bundle technique. Arthroscopy. 2002;18:E19.

13. Camarda L, Grassedonio E, Lauria M, Midiri M, D'Arienzo M. How to avoid collision between $P C L$ and $M C L$ femoral tunnels during a simultaneous reconstruction. Knee Surg Sports Traumatol Arthrosc. 2014; In press.

14. LaPrade RF, Engebretsen AH, Ly TV, Johansen S, Wentorf FA, Engebretsen L. The anatomy of the medial part of the knee. J Bone Joint Surg Am. 2007;89:2000-10.

15. Liu F, Yue B, Gadikota HR, Kozanek M, Liu W, Gill TJ, Rubash HE, Li G. Morphology of the medial collateral ligament of the knee. J Orthop Surg Res. 2010;5:69.

16. Hughston JC, Eilers AF. The role of the posterior oblique ligament in repairs of acute medial (collateral) ligament tears of the knee. J Bone Joint Surg Am. 1973;55:923-40.

17. Liu X, Feng $H$, Zhang $H$, Hong L, Wang XS, Zhang J, Shen JW. Surgical treatment of subacute and chronic valgus instability in multiligamentinjured knees with superficial medial collateral ligament reconstruction using Achilles allografts: a quantitative analysis with a minimum 2-year follow-up. Am J Sports Med. 2013:41:1044-50.

18. Smyth MP, Koh JL. A review of surgical and nonsurgical outcomes of medial knee injuries. Sports Med Arthrosc. 2015;23:e15-22.

19. Van den Bogaerde JM, Shin E, Neu CP, Marder RA. The superficial medial collateral ligament reconstruction of the knee: effect of altering graft length on knee kinematics and stability. Knee Surg Sports Traumatol Arthrosc. 2011;19 Suppl 1:S60-8 